\title{
Comparison of the Distribution Pattern of 21-Gene Recurrence Score between Mucinous Breast Cancer and Infiltrating Ductal Carcinoma in Chinese Population: A Retrospective Single-Center Study
}

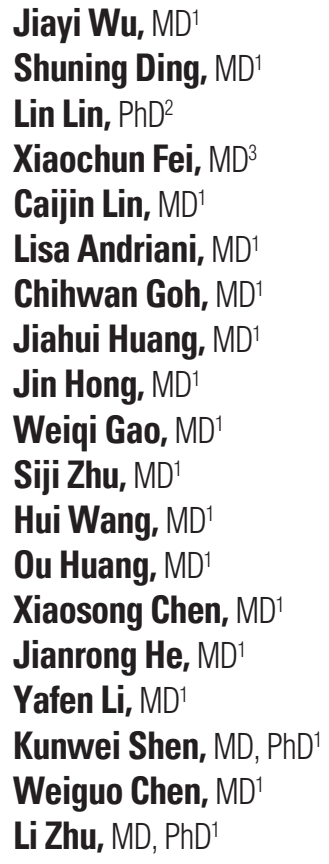

*A list of author's affiliations appears at the end of the paper.

Correspondence: $\mathrm{Li}$ Zhu, MD, PhD

Comprehensive Breast Health Center,

Ruijin Hospital, Shanghai Jiao Tong University

School of Medicine, No.197 Ruijin Er Road,

Shanghai 200025, China

Tel: 86-21-6437-0045

Fax: 86-21-6415-6886

E-mail: zhuli8@yeah.net

Co-correspondence: Weiguo Chen, MD Comprehensive Breast Health Center, Ruijin Hospital, Shanghai Jiao Tong University School of Medicine, No.197 Ruijin Er Road, Shanghai 200025, China

Tel: 86-21-6437-0045

Fax: 86-21-6415-6886

E-mail: cwg-dr@hotmail.com

Received July 8, 2019

Accepted January 23, 2020

Published Online January 28, 2020

*Jiayi Wu and Shuning Ding contributed equally to this work.

\begin{abstract}
Purpose
This retrospective study aimed to evaluate the distribution pattern and prognostic value of 21-gene recurrence score (RS) in Chinese patients with mucinous breast cancer (MC) and compared with infiltrating ductal carcinoma (IDC).
\end{abstract}

\section{Materials and Methods}

Patients diagnosed with MC or IDC from January 2010 to January 2017 were retrospectively recruited. Reverse transcriptase-polymerase chain reaction assay of 21 genes was conducted to calculate the RS. Univariate and multivariate analyses were performed to assess the association between RS and clinicopathological factors. Survival outcomes including disease-free survival (DFS) and overall survival (OS) were estimated by Kaplan-Meier method and compared by log-rank test.

\section{Results}

The MC cohort included 128 patients and the IDC cohort included 707 patients. The proportions of patients with a low (RS $<18)$, intermediate $(18-30)$, or high risk (RS $>30$ ) were $32.0 \%, 48.4 \%$, and $19.5 \%$ in MC cohort, and $26.9 \%, 46.8 \%$ and $26.3 \%$ in IDC cohort. The distribution of RS varied significantly according to different Ki-67 index and molecular subtype in both cohorts. Moreover, the receipt of chemotherapy was associated with RS in both cohorts. Among patients with MC, tumor stage was related to the DFS $(p=0.040)$. No significant differences in DFS and OS were found among MC patients in different RS risk groups (OS, $p=0.695 ;$ DFS, $p=0.926$ ).

\section{Conclusion}

RS was significantly related to Ki-67 index and molecular subtypes in MC patients, which is similar in IDC patients. However, RS was not able to predict DFS and OS in patients with MC.

\section{Key words}

Mucinous mammary malignancy, Multigene assay, Prognosis 


\section{Introduction}

Over recent years, facilitated by substantial advances in high-throughput analyses, multigene expression profiling has gained rapid development and become powerful tools for predicting the risk of relapse and mortality in patients with breast cancer.

The 21-gene recurrence score (RS) assay (Oncotype Dx, Genomic Health, Redwood City, CA) was calculated based on the results of a reverse transcriptase-polymerase chain reaction (RT-PCR) assay of 21 prospectively selected genes in paraffin-embedded tumor tissues [1]. Previous studies have validated the ability of 21-gene assay to provide additional prognostic information beyond classical clinicopathological features in patients with estrogen receptor (ER)-positive, human epidermal growth factor receptor-2 (HER2)-negative and node-negative invasive breast cancer treated with adjuvant tamoxifen or anastrozole alone [1,2]. Additionally, 21-gene assay was validated to be capable of predicting benefit from adjuvant chemotherapy in node-negative and nodepositive patients according to the analyses of the NSABP B-20 and SWOG-8814 trials [3,4]. As a result of its excellent prognostic and predictive ability, 21-gene RS has been widely used by clinicians to help predict outcomes and make therapeutic decisions.

Mucinous breast cancer (MC) is a rare subtype of invasive breast cancer, with an incidence of $1 \%-6 \%$ in all primary breast cancer [5-10]. Prior series indicated that the majority of MC were ER-positive, HER2-negative tumors without node metastasis [11-14], which matches the main criteria of 21-gene genomic test. However, the retrospective studies aimed at validating the prognostic value of RS did not distinguish among histological subtypes and the study cohorts were mainly compromised by infiltrating ductal carcinoma (IDC) $[1,3]$. Given the relatively low incidence of MC, no study to date has explicated the prognostic significance of RS in MC.

Therefore, the objective of the current study was to evaluate the distribution pattern and prognostic value of RS in Chinese patients with MC compared to IDC.

\section{Materials and Methods}

\section{Study population and follow-up}

Patients who were diagnosed with MC or IDC from January 2010 to January 2017 at Shanghai Jiao Tong University Affiliated Ruijin Hospital were retrospectively reviewed. The detailed inclusion criteria are as follows: (1) female sex; (2) $\mathrm{MC}$ or IDC proven by core needle biopsy or open excision biopsy; (3) underwent surgical procedure; (4) ER-positive and HER2-negative tumors; (5) available fixed, paraffin-embedded tumor tissue for RS test; (6) complete clinical and follow-up data. Women with T1a or T4 tumor, metastatic breast cancer, or treated with neoadjuvant treatment were excluded.

Patients were divided into MC cohort and IDC cohort according to pathology. The baseline clinicopathological characteristics were obtained from the prospectively maintained Shanghai Jiao Tong University Breast Cancer Database (SJTU-BCBD). Demographic and clinical data including age, type of surgery, pathology, tumor grade, tumor size, progesterone receptor (PR) status, and Ki-67 index were collected. Treatment and follow-up information was obtained by phone calls and/or outpatient medical record.

Disease-free survival (DFS) was defined as time from diagnosis to the earliest of disease recurrence including local/ regional recurrence and distant metastasis in any site, second primary cancer, or death of any cause. Overall survival (OS) was defined as time from diagnosis to death as a result of any cause. The last follow-up was conducted in March 2018.

The current study has been approved by the Ethical Committees of Shanghai Ruijin Hospital. The results of this study do not affect the treatment decision of any patient included.

\section{Sample preparation and RS calculation}

The methods of sample preparation and RS calculation were similar to that previously described $[15,16]$. Briefly, hematoxylin and eosin-stained slides were reviewed by pathologists to make sure that the paraffin section used for 21-gene RS analysis contained sufficient tumor tissue. After RNA extraction and DNase I treatment, the RT-PCR reactions in 96 well plates were performed using Applied Biosystems (Foster City, CA) 7500 Real-Time PCR System. Expression of 16 cancer-related genes was measured in triplicate and normalized relative to a set of five reference genes. The recurrence score, scaled from 0 to 100, was then calculated for each patient using the algorithm previously described in detail [1]. Patients were classified by the pre-specified cutoff points into low-risk ( $R S<18)$, intermediate-risk (18-30), and high-risk (RS $>30)$ groups.

\section{Pathological assessment}

The immunohistochemistry (IHC) assessment of biomarkers, including ER, PR, Ki-67, and C-erb B2, were conducted according to previously described protocols $[17,18]$. ER/PR status was defined as positive if there are at least $1 \%$ positive tumor nuclei in the sample according to the recommendation 
Table 1. Clinicopathological characteristics and treatment information of the included patients

\begin{tabular}{|c|c|c|c|}
\hline Characteristic & $\mathrm{MC}(\mathrm{n}=128)$ & IDC $(n=707)$ & p-value \\
\hline \multicolumn{4}{|l|}{ Age (yr) } \\
\hline$<55$ & $43(33.6)$ & $349(49.4)$ & 0.001 \\
\hline$\geq 55$ & $85(66.4)$ & $358(50.6)$ & \\
\hline \multicolumn{4}{|l|}{ Surgery } \\
\hline Mastectomy & $77(60.2)$ & $399(56.4)$ & 0.434 \\
\hline BCS & $51(39.8)$ & $308(43.6)$ & \\
\hline \multicolumn{4}{|l|}{ T category } \\
\hline $\mathrm{T} 1$ & $70(54.7)$ & $494(69.9)$ & 0.001 \\
\hline $\mathrm{T} 2-3$ & $58(45.3)$ & $213(30.1)$ & \\
\hline \multicolumn{4}{|l|}{ Node status } \\
\hline Negative & $120(93.8)$ & $614(86.8)$ & 0.028 \\
\hline Positive & $8(6.2)$ & $93(13.2)$ & \\
\hline \multicolumn{4}{|l|}{ PR } \\
\hline Positive & $109(85.2)$ & $599(84.7)$ & 0.900 \\
\hline Negative & $19(14.8)$ & $108(15.3)$ & \\
\hline \multicolumn{4}{|l|}{ Ki-67 group } \\
\hline Low & $59(46.1)$ & $398(56.3)$ & \\
\hline High & $69(53.9)$ & 309 (43.7) & 0.033 \\
\hline \multicolumn{4}{|l|}{ Molecular subtype } \\
\hline Luminal A-like & $51(39.8)$ & $297(42.0)$ & 0.648 \\
\hline Luminal B-like & $77(60.2)$ & $410(58.0)$ & \\
\hline \multicolumn{4}{|l|}{ Chemotherapy } \\
\hline Yes & 17 (13.3) & $381(53.9)$ & $<0.001$ \\
\hline No & $111(86.7)$ & $326(46.1)$ & \\
\hline \multicolumn{4}{|l|}{ Radiation } \\
\hline Yes & $48(37.5)$ & $320(45.3)$ & 0.104 \\
\hline No & $80(62.5)$ & $387(54.7)$ & \\
\hline
\end{tabular}

Values are presented as number (\%). MC, mucinous breast cancer; IDC, infiltrating ductal carcinoma; BCS, breast-conserving surgery; PR, progesterone receptor.

of the American Society of Clinical Oncology (ASCO) and College of American Pathologists (CAP) [19]. IHC assessments of Ki-67 were made from paraffin-embedded tumor samples and MIB-1 (mouse monoclonal, Dako) was the antibody used for IHC test. To calculated the Ki-67 index, the cell distribution over the whole slice was reviewed at first. If Ki-67 expression was uniformly distributed over the entire slide, 500-2,000 cells were chosen from different microscope views; otherwise, 1,000 cells were equally counted in both hotspot and negative areas in slice. Ki-67 index was characterized as the proportion of positively nuclear staining cells among at least 1,000 tumor cells in the area counted and recorded as mean percentage of positive cells. In this retrospective analysis, the median $\mathrm{Ki}-67$ index in each cohort was set as the cut-off to distinguish between high and low Ki-67 index. HER2 status was considered as negative if it scored 0 to $1+$ by IHC. Fluorescent in situ hybridization was performed to all IHC $2+$ and $3+$ to determine the HER2 gene amplification.

Patients were classified into luminal A and luminal B according to the IHC-based surrogate subtypes. Based on international recommendations and the specific laboratory protocols at department of pathology in Ruijin Hospital, the surrogate subtype classifications were described as follows: (1) luminal A-like, HER2 negative, PR positive, and low Ki-67 index; (2) luminal B-like, HER2 negative, high Ki-67 index, or PR negative.

\section{Statistical analysis}

The Pearson chi-square or Fisher exact test when necessary was performed to compare the distribution of clinicopathological characteristics in MC cohort versus IDC cohort and to assess the distribution of RS in patients with different clinicopathological characteristics. Multi-variable logistic regression model was used to determine independent factors 
Table 2. Distribution of RS by clinicopathological factors in MC cohort and IDC cohort

\begin{tabular}{|c|c|c|c|c|c|c|c|c|}
\hline \multirow[b]{2}{*}{ Characteristic } & \multicolumn{4}{|c|}{ MC cohort (n=128) } & \multicolumn{4}{|c|}{ IDC cohort ( $\mathrm{n}=707)$} \\
\hline & $\begin{array}{l}\text { Low risk } \\
(\mathrm{RS}<18)\end{array}$ & $\begin{array}{l}\text { Intermediate } \\
\text { risk (RS } 18 \text { to 30) }\end{array}$ & $\begin{array}{l}\text { High risk } \\
(\mathrm{RS} \geq 31)\end{array}$ & p-value & $\begin{array}{l}\text { Low risk } \\
(\mathrm{RS}<18)\end{array}$ & $\begin{array}{l}\text { Intermediate } \\
\text { risk (RS } 18 \text { to 30) }\end{array}$ & $\begin{array}{c}\text { High risk } \\
(\mathrm{RS} \geq 31)\end{array}$ & p-value \\
\hline \multicolumn{9}{|l|}{ Age (yr) } \\
\hline$<55$ & $9(20.9)$ & $23(53.5)$ & $11(25.6)$ & 0.132 & $88(25.2)$ & $163(46.7)$ & $98(28.1)$ & 0.465 \\
\hline$\geq 55$ & $32(37.6)$ & $39(45.9)$ & $14(16.5)$ & & $102(28.5)$ & $168(46.9)$ & $88(24.6)$ & \\
\hline \multicolumn{9}{|l|}{ Surgery } \\
\hline Mastectomy & $27(35.1)$ & $36(46.8)$ & $14(18.2)$ & 0.704 & $115(28.8)$ & $183(45.9)$ & $101(25.3)$ & 0.403 \\
\hline BCS & $14(27.5)$ & $26(51.0)$ & $11(21.6)$ & & $75(24.4)$ & $148(48.1)$ & 85 (27.6) & \\
\hline \multicolumn{9}{|l|}{$T$ category } \\
\hline $\mathrm{T} 1$ & $20(28.6)$ & $38(54.3)$ & $12(17.1)$ & 0.349 & $145(29.4)$ & $234(47.4)$ & $115(23.3)$ & 0.008 \\
\hline T2-3 & $21(36.2)$ & $24(41.4)$ & $13(22.4)$ & & $45(21.1)$ & $97(45.5)$ & $71(33.3)$ & \\
\hline \multicolumn{9}{|l|}{ Node status } \\
\hline Negative & $38(31.7)$ & $58(48.3)$ & $24(20.0)$ & 1.000 & $171(27.9)$ & $282(45.9)$ & $161(26.2)$ & 0.293 \\
\hline Positive & $3(37.5)$ & $4(50.0)$ & $1(12.5)$ & & $19(20.4)$ & $49(52.7)$ & $25(26.9)$ & \\
\hline \multicolumn{9}{|l|}{ PR } \\
\hline Positive & $37(33.9)$ & $52(47.7)$ & $20(18.3)$ & 0.437 & $180(30.1)$ & $284(47.4)$ & $135(22.5)$ & $<0.001$ \\
\hline Negative & $4(21.1)$ & $10(52.6)$ & $5(26.3)$ & & $10(9.3)$ & 47 (43.5) & $51(47.2)$ & \\
\hline \multicolumn{9}{|l|}{ Ki-67 } \\
\hline Low $(<10,<15)$ & $26(44.1)$ & $24(40.7)$ & $9(15.3)$ & 0.028 & $111(32.2)$ & 178 (51.6) & $56(16.2)$ & $<0.001$ \\
\hline $\operatorname{High}(\geq 10, \geq 15)$ & $15(21.7)$ & $38(55.1)$ & $16(23.2)$ & & $79(21.8)$ & $153(42.3)$ & $130(35.9)$ & \\
\hline \multicolumn{9}{|l|}{ Subtype } \\
\hline Luminal A-like & $23(45.1)$ & $21(41.2)$ & $7(13.7)$ & 0.035 & $106(35.7)$ & $150(50.5)$ & $41(13.8)$ & $<0.001$ \\
\hline Luminal B-like & $18(23.4)$ & $41(53.2)$ & $18(23.4)$ & & $84(20.5)$ & $181(44.1)$ & $145(35.4)$ & \\
\hline
\end{tabular}

Values are presented as number (\%). RS, recurrence score; MC, mucinous breast cancer; IDC, infiltrating ductal carcinoma; $\mathrm{BCS}$, breast-conserving surgery; PR, progesterone receptor.

related to RS groups. Kaplan-Meier analyses were conducted to estimate the rate, and log-rank tests were performed to compare the survival curves. Multivariate Cox proportional hazards models were used to identify the independent prognostic factor for DFS and OS. All Statistical analyses were conducted with the statistical package STATA ver. 14.0 for Windows 10 (College Station, TX). A p-value of less than 0.05 (two-sided) was considered to indicate a significant result.

\section{Ethical statement}

All procedures performed in studies involving human participants were in accordance with the ethical standards of the independent Ethical Committee/Institutional Review Board of Ruijin Hospital (14411950200) and with the 1964 Helsinki declaration and its later amendments or comparable ethical standards. Informed consent was exempted due to the retrospective format of this study.

\section{Results}

\section{Clinicopathological features}

The MC cohort included 128 patients, with 85 patients aged 50 and above (66.4\%), while the IDC cohort included 707 patients, with 358 patients aged 50 and above $(50.6 \%)$. Patients with MC were significantly older than patients with IDC $(p=0.001)$. The baseline clinicopathological features of both cohorts were outlined in Table 1.

Compared with IDC, patients with MC had less T1 tumors (MC vs. IDC, $54.7 \%$ vs. $69.9 \%$; $\mathrm{p}<0.001$ ) and less lymph node involvement at diagnosis (MC vs. IDC, $6.2 \%$ vs. $13.2 \%$; $\mathrm{p}=0.028)$. In MC cohort, the mean and median Ki-67 index were $11.9 \%$ and $10 \%$, while in IDC cohort, the mean and median Ki-67 index were $19.3 \%$ and $15 \%$. The percentage of PR-positive tumors was similar between patients with MC and IDC ( $85.2 \%$ vs. $84.7 \%, \mathrm{p}=0.900)$. With respect to the molecular subtype, there was no significant difference between the two cohorts $(\mathrm{p}=0.648)$, with $39.8 \%$ of luminal A-like 


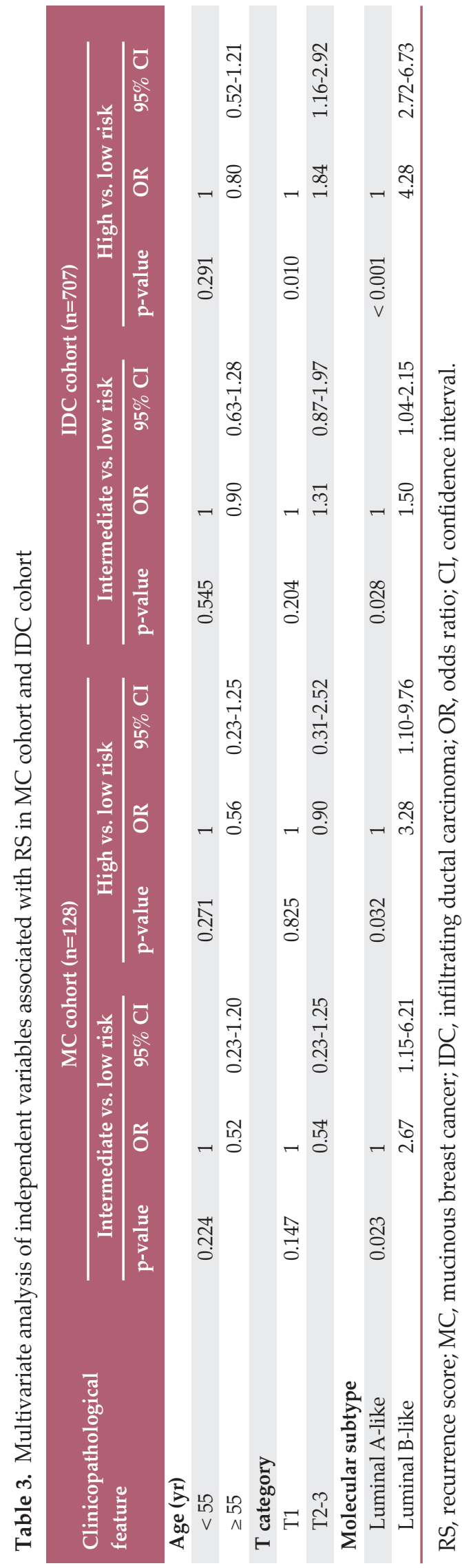

tumors in MC cohort and $42 \%$ of luminal B-like tumors in IDC cohort.

In our series, the percentage of patients underwent breastconserving surgery and received radiation was similar in MC cohort and IDC cohort. However, chemotherapy was significantly less common in patients with MC $(13.3 \%)$ than in patients with IDC $(53.9 \%, \mathrm{p}<0.001)$. All patients received appropriate endocrine treatment.

\section{Distribution of RS}

With regard to RS category, the proportions of patients with low-, intermediate-, and high-risk RS were $32.0 \%$, 48.4\%, and $19.5 \%$ in MC cohort, $26.9 \%, 46.8 \%$, and $26.3 \%$ in IDC cohort. The distribution of RS according to different clinicopathological factors are shown in Table 2.

In MC cohort, RS varied significantly according to different Ki-67 index ( $\mathrm{p}=0.028)$ and molecular subtypes $(\mathrm{p}=0.035)$. Patients with high Ki-67 index and luminal B subtype tumors were more likely to have high-risk RSs. Similarly, RS was correlated to different $\mathrm{Ki}-67$ index and molecular subtype in IDC cohort $(p<0.001)$. In IDC cohort, tumor size was associated with RS group as well $(p<0.001)$. The percentage of patients having high-risk RS is higher in patients with $\mathrm{T} 2$ or T3 tumors (33.3\%) than in patients with T1 tumors (23.3\%).

Multivariate regression analyses including age, pathological $\mathrm{T}$ category, and molecular subtype revealed that molecular subtype was independently related to the distribution of RS in both MC and IDC cohorts (Table 3). In MC cohort, luminal B-like tumors were significantly associated with higher likelihood of having intermediate-risk RSs (hazard ratio [HR], 2.67; 95\% confidence interval [CI], 1.15 to 6.21; $\mathrm{p}=0.023$ ) and high-risk RSs (HR, 3.28; 95\% CI, 1.10 to 9.76; $\mathrm{p}=0.032$ ) compared to low-risk RSs. Likewise, patients with luminal B-like tumors in IDC cohort were more likely to have intermediate-risk RSs (HR, 1.50; 95\% CI, 1.04 to 2.15 ; $\mathrm{p}=0.028$ ) and high-risk RSs (HR, 4.28; 95\% CI, 2.72 to $6.73 \mathrm{p}<0.001)$ rather than low-risk RSs. It was also indicated that T2 or T3 tumors in IDC cohort were more likely to have high-risk RSs compared to low-risk RSs (HR, 1.84; 95\% CI, 1.16 to 2.92; $\mathrm{p}=0.010$ ).

As for treatment, the receipt of chemotherapy was corelated with RS in MC cohort $(\mathrm{p}=0.019)$ and in IDC cohort $(\mathrm{p}<$ 0.001). The percentage of patients receiving chemotherapy in the low-, intermediate-, and high-risk RS group was $2.4 \%$, $16.4 \%$, and $24.0 \%$ in MC cohort, respectively, while the percentage was $21.6 \%, 58.3 \%$, and $79.0 \%$ in IDC cohort.

\section{Univariate and multivariate analyses of DFS and OS}

After a median follow-up of 26.4 months (range, 2.9 to 105.4 months), there were nine DFS events and two OS 
Table 4. Univariate prognostic analyses of DFS and OS in MC cohort $(n=128)$ and IDC cohort $(n=707)$

\begin{tabular}{|c|c|c|c|c|c|c|c|c|}
\hline \multirow{3}{*}{ Characteristic } & \multicolumn{4}{|c|}{ MC } & \multicolumn{4}{|c|}{ IDC } \\
\hline & \multicolumn{2}{|l|}{ DFS } & \multicolumn{2}{|l|}{ OS } & \multicolumn{2}{|l|}{ DFS } & \multicolumn{2}{|l|}{ OS } \\
\hline & Survival rate $(\%)$ & p-value & Survival rate $(\%)$ & p-value & Survival rate (\%) & p-value & Survival rate $(\%)$ & p-value \\
\hline \multicolumn{9}{|l|}{ Age (yr) } \\
\hline$<55$ & 91.2 & 0.470 & 100 & 0.381 & 94.0 & 0.996 & 98.9 & 0.030 \\
\hline$\geq 55$ & 95.4 & & 95.6 & & 94.7 & & 96.9 & \\
\hline \multicolumn{9}{|l|}{ Surgery } \\
\hline Mastectomy & 90.9 & 0.842 & 98.7 & 0.457 & 94.2 & 0.815 & 97.0 & 0.174 \\
\hline BCS & 96.1 & & 98.0 & & 94.5 & & 99.0 & \\
\hline \multicolumn{9}{|l|}{$\mathrm{T}$ category } \\
\hline $\mathrm{T} 1$ & 97.1 & 0.040 & 100 & 0.123 & 95.7 & 0.006 & 98.2 & 0.303 \\
\hline $\mathrm{T} 2-3$ & 87.9 & & 96.6 & & 91.1 & & 97.2 & \\
\hline \multicolumn{9}{|l|}{ Nodal status } \\
\hline Negative & 92.5 & 0.518 & 98.3 & 0.741 & 93.8 & 0.389 & 98.9 & 0.868 \\
\hline Positive & 100 & & 100 & & 97.8 & & 97.7 & \\
\hline \multicolumn{9}{|l|}{ PR } \\
\hline Positive & 92.7 & 0.705 & 98.2 & 0.525 & 95.0 & 0.073 & 97.8 & 0.836 \\
\hline Negative & 94.7 & & 100 & & 90.7 & & 98.1 & \\
\hline \multicolumn{9}{|l|}{ Ki67 } \\
\hline Low & 91.5 & 0.979 & 100 & 0.135 & 95.7 & 0.038 & 98.5 & 0.196 \\
\hline High & 94.2 & & 97.1 & & 92.6 & & 97.1 & \\
\hline \multicolumn{9}{|l|}{ Subtype } \\
\hline Luminal A-like & 92.2 & 0.936 & 100 & 0.215 & 96.3 & 0.045 & 98.7 & 0.153 \\
\hline Luminal B-like & 93.5 & & 97.4 & & 92.9 & & 97.3 & \\
\hline \multicolumn{9}{|l|}{ Chemotherapy } \\
\hline Yes & 100 & 0.263 & 100 & 0.556 & 93.4 & 0.123 & 97.6 & 0.381 \\
\hline No & 91.9 & & 98.2 & & 95.4 & & 98.2 & \\
\hline \multicolumn{9}{|l|}{ Radiation } \\
\hline Yes & 97.9 & 0.312 & 100 & 0.378 & 95.9 & 0.205 & 99.1 & 0.144 \\
\hline No & 90.0 & & 97.5 & & 93.0 & & 96.9 & \\
\hline \multicolumn{9}{|l|}{ RS category } \\
\hline Low & 92.7 & 0.926 & 97.6 & 0.695 & 96.8 & 0.023 & 98.9 & 0.296 \\
\hline Intermediate & 95.2 & & 98.4 & & 95.8 & & 98.5 & \\
\hline High & 88.0 & & 100 & & 89.2 & & 95.7 & \\
\hline
\end{tabular}

DFS, disease-free survival; OS, overall survival; MC, mucinous breast cancer; IDC, invasive ductal carcinoma; BCS, breastconserving surgery; PR, progesterone receptor; RS, recurrence score.

events in MC cohort while 40 DFS events and 15 OS events in IDC cohort.

With respect to DFS, the results of univariate analyses showed that pathologic $\mathrm{T}$ category was the only factor significantly associated with DFS among patients with MC $(\mathrm{p}=0.040)$. The 3-year DFS rates for patients with T1 tumors and patients with T2-3 tumors was $97.1 \%$ and $87.9 \%$, respectively (Table 4). According to multivariate Cox model, the differences in DFS between pathologic $\mathrm{T}$ categories were marginally significant $(p=0.060)$. No significant correlations were founded between RS category and DFS in both univariate and multivariate analyses (Table 4, Fig. 1A). The 3-year
DFS rates for patients with low-, intermediate-, and high-risk RS were $92.7 \%, 95.2 \%$, and $88.0 \%$ in MC cohort, respectively (Table 4). While in the IDC cohort, the results of univariate analyses indicated that pathologic $T$ category $(p=0.006)$, Ki-67 index $(p=0.038)$, molecular subtype ( $p=0.045)$, and RS category $(p=0.023)$ were all significantly associated with DFS (Table 4). According to multivariate analyses, only pathologic T category was identified as an independent prognostic factor for DFS (HR, 2.03; 95\% CI, 1.08 to 3.81; $\mathrm{p}=0.028$ ). The 3 -year DFS rates for patients with low-, intermediate-, and high-risk RS were $96.8 \%, 95.8 \%$, and $89.2 \%$ in IDC cohort, respectively $(\mathrm{p}=0.023)$ (Table 4 , Fig. $2 \mathrm{~A})$ but $\mathrm{RS}$ category 


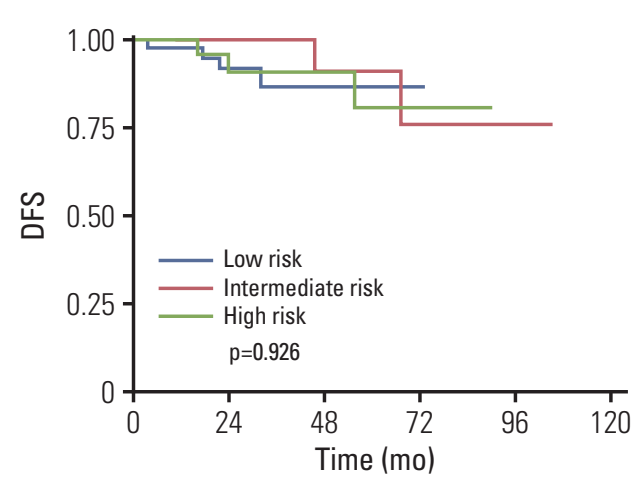

A

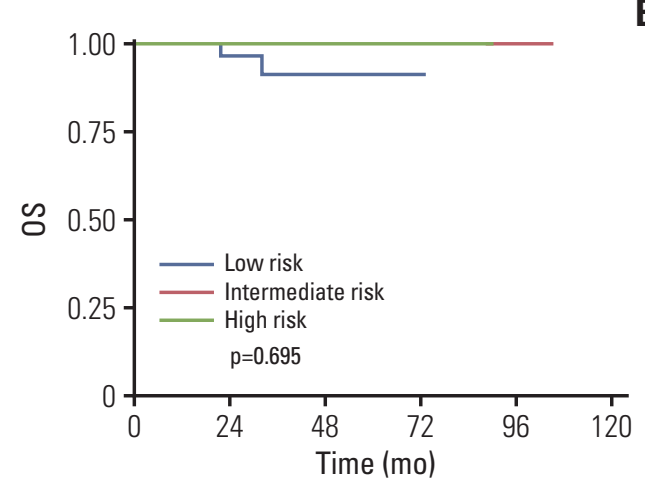

B

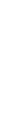

Fig. 1. Kaplan-Meier survival curves in mucinous breast cancer patients according to different recurrence score risk groups. Disease-free survival (DFS) (A) and overall survival (OS) (B).

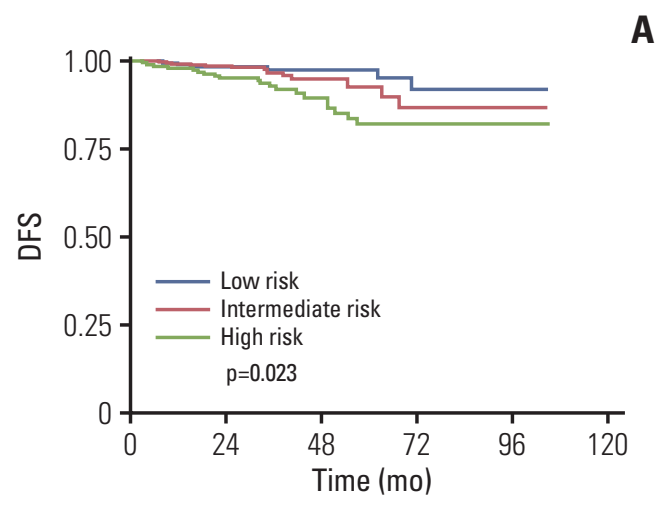

A

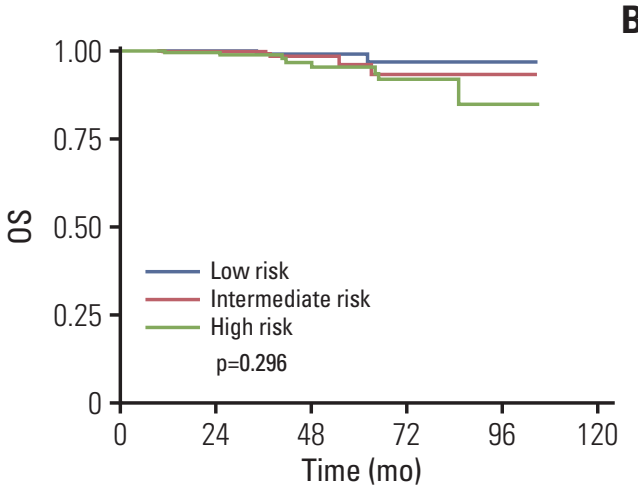

Fig. 2. Kaplan-Meier survival curves in invasive ductal carcinoma patients according to different recurrence score risk groups. Disease-free survival (DFS) (A) and overall survival (OS) (B).

failed to predict DFS according to multivariate analyses ( $\mathrm{p}=0.160$; intermediate risk vs. low risk: $\mathrm{HR}, 1.42 ; 95 \% \mathrm{CI}$, 0.54 to 3.37 ; high risk vs. low risk: $\mathrm{HR}, 2.31 ; 95 \% \mathrm{CI}, 0.90$ to $5.90)$.

With respect to OS, neither different pathologic $\mathrm{T}$ category ( $\mathrm{p}=0.123$ ) nor RS category ( $\mathrm{p}=0.695$ ) was associated with OS among patients with $\mathrm{MC}$, according to the results of univariate analyses (Table 4, Fig. 1B). This may be due to limited number of events. The 3-year OS rates for patients with low-, intermediate-, and high-risk RS were $97.6 \%, 98.4 \%$, and $100 \%$, respectively (Table 4). Similarly, in IDC cohort, neither different pathological $\mathrm{T}$ category ( $\mathrm{p}=0.303$ ) nor RS category $(p=0.296)$ can significantly predict OS according to univariate analyses (Table 4, Fig. 2B). The 3-year OS rates for patients with low-, intermediate-, and high-risk RS were $98.9 \%$, $98.5 \%$, and $95.7 \%$, respectively (Table 4 ).

\section{Discussion}

The current study evaluated the distribution pattern and prognostic significance of 21-gene RS in a cohort of 128 Chinese $\mathrm{MC}$ patients and assessed the factors associated with RS in a cohort of 707 Chinese IDC patients for comparison. RS has been proved to have both prognostic and predictive power in ER-positive, HER2-negative, and node-negative patients with invasive breast cancer [1,3]. MC met the criteria of 21-gene test. But up to now, the utility of RS has not been studied in MC cohort. To our knowledge, our study represents the first study focused on the distribution pattern and prognostic value of 21-gene RS in MC.

Based on the traditional RS category, the proportion of patients having high-risk RS was $19.5 \%$ in current MC cohort, lower than the percentage of $27 \%$ and $25.2 \%$ observed in validation studies of RS conducted in patients from 
NSABP B-14 and B-20 trial [1,3]. Whereas, the proportion of patients with high-risk RS was $26.3 \%$ in current IDC cohort, which was in line with the percentage of high-risk RS group mentioned above. The disparity in the distribution of RS risk groups among different pathological subtypes was in concordance with a previous finding, which indicated that patients with MC had a lower likelihood of having a highrisk RS compared with patients with IDC [20]. Likewise, invasive lobular carcinoma, in which ER-positive and HER2negative tumors were the majority, mainly had low-risk RS $(60 \%)$ and intermediate-risk RS $(38 \%)$. Consistent with the previous study, the predominance of low- and intermediaterisk RS in MC cohort was also found in our study, suggesting better prognosis of MC.

In our series, the Ki-67 index and molecular subtype was associated with the distribution of RS, and molecular subtype can independently predict RS in both MC and IDC cohorts. In high Ki-67 group, the proportion of patients having highrisk RSs was $23.2 \%$ in MC cohort and $35.4 \%$ in IDC cohort, which were significantly higher than those in low Ki-67 group. In concordance with our results, Ki-67 index was also reported to be associated with RS in WSG Plan B trial [21]. PR status was associated with RS in current IDC cohort. Likewise, PR status was also defined as a predictor for RS according to the previous analyses among patients in Plan B and NASBP B20 $[3,21]$. In current MC cohort, the proportion of patients categorized into low-, intermediate-, and high-risk groups were $33.9 \%, 47.7 \%$, and $18.3 \%$ in patients with PRpositive tumors, while $21.1 \%, 52.6 \%$, and $26.3 \%$ in those with PR-negative tumors. RS distributed differently according to PR status but the difference did not meet statistical significance, which may be resulted from the insufficient sample size. Moreover, both PR status and Ki-67 index were identified as independent predictors for RS in our previous study in 980 Chinese estrogen receptor-positive, HER2-negative breast cancer patients [15]. Multivariate analyses in our current study showed that molecular subtype defined by Ki-67 and PR level was an independent predictor for RS in both $\mathrm{MC}$ and IDC cohorts. Luminal B-like tumors were more likely to have high-risk RSs. These facts indicated that routine clinical and pathologic parameters might be of help in predicting RS.

The DFS rates and OS rates were relatively high in current study, with only nine DFS events and two OS events with 3-year estimated DFS rates of $92.7 \%, 95.2 \%$, and $88.7 \%$, and 3-year estimated OS rates of $97.6 \%, 98.4 \%$, and $100 \%$ for low, intermediate, and high RS risk categories in MC cohort. However, the differences of survival outcomes among different RS risk groups were not statistically significant. Subgroup analyses conducted in patients without chemotherapy also confirmed this finding.

The possible reason for the negative results may lie in the favorable clinicopathological features and good prognosis in MC cohort. Previous study conducted in 11,400 patients with MC indicated that patients with pure MC had better outcomes than IDC patients [22], with 10-, 15-, and 20-year survival rates were $89 \%, 85 \%$, and $81 \%$, respectively, compared to $82 \%$ (5 years), $72 \%$ (10 years), 66\% (15 years), and $62 \%$ (20 years) for IDC. Another possible reason may be that the follow-up was not long enough and the sample size was small to observe the differences in survival.

According to our analyses, the percentage of patients of receiving chemotherapy was higher in the high-risk RS group compared with low- and intermediate-RS group, but the survival outcomes were not significantly different between patients with or without chemotherapy in each RS group ( $p>0.05)$. The potential benefit of chemotherapy in MC cohort may need longer follow-up and further investigation.

Pathological tumor size remained the significant predictor for DFS according to univariate analyses and was marginally significantly associated with DFS according to multivariate analyses which was possibly caused by the relatively short follow-up and limited number of events, indicating that traditional TNM (tumor size, nodal status, metastasis) classification system remained fundamental for tumor staging and prognosis prediction in patients with MC. Traditional clinicopathological factors that described tumor burden were of great value in predicting outcomes and making therapeutic decisions in MC, which were dominated by ER-positive, HER2-negative tumors. Based on the current study, RS did not possess a distinct advantage over tumor burden in $\mathrm{MC}$ cohort.

In conclusion, patients with MC tend to have low- and intermediate-risk RS. Molecular subtype defined by PR status and Ki-67 index can independently predict high-risk RS among patients with MC, as well as in patients with IDC. Further research is necessary to evaluate the use of RS in predicting outcomes among patients with MC.

\section{Conflicts of Interest}

Conflict of interest relevant to this article was not reported.

\section{Acknowledgments}

This study was supported by National Natural Science Foundation of China (grant number: 81572581 and 81772797), Technology Innovation Act Plan of Shanghai Municipal Science and Technology Commission (grant number: 14411950200, 14411950201, 16411966900) and Grant of Shanghai municipal commission of health and family planning (201840323). The funders had no role in study design, data collection and analysis, decision to publish, or preparation of the manuscript. 


\section{Author Details}

${ }^{1}$ Comprehensive Breast Health Center, Departments of ${ }^{2}$ Clinical
Laboratory and ${ }^{3}$ Pathology, Ruijin Hospital, Shanghai Jiao Tong University School of Medicine, Shanghai, China

\section{References}

1. Paik S, Shak S, Tang G, Kim C, Baker J, Cronin M, et al. A multigene assay to predict recurrence of tamoxifen-treated, node-negative breast cancer. N Engl J Med. 2004;351:2817-26.

2. Dowsett M, Cuzick J, Wale C, Forbes J, Mallon EA, Salter J, et al. Prediction of risk of distant recurrence using the 21-gene recurrence score in node-negative and node-positive postmenopausal patients with breast cancer treated with anastrozole or tamoxifen: a TransATAC study. J Clin Oncol. 2010;28: 1829-34.

3. Paik S, Tang G, Shak S, Kim C, Baker J, Kim W, et al. Gene expression and benefit of chemotherapy in women with nodenegative, estrogen receptor-positive breast cancer. J Clin Oncol. 2006;24:3726-34.

4. Albain KS, Barlow WE, Shak S, Hortobagyi GN, Livingston RB, Yeh IT, et al. Prognostic and predictive value of the 21gene recurrence score assay in postmenopausal women with node-positive, oestrogen-receptor-positive breast cancer on chemotherapy: a retrospective analysis of a randomised trial. Lancet Oncol. 2010;11:55-65.

5. Komaki K, Sakamoto G, Sugano H, Morimoto T, Monden Y. Mucinous carcinoma of the breast in Japan. A prognostic analysis based on morphologic features. Cancer. 1988;61:98996.

6. Andre S, Cunha F, Bernardo M, Meneses e Sousa J, Cortez F, Soares J. Mucinous carcinoma of the breast: a pathologic study of 82 cases. J Surg Oncol. 1995;58:162-7.

7. Diab SG, Clark GM, Osborne CK, Libby A, Allred DC, Elledge RM. Tumor characteristics and clinical outcome of tubular and mucinous breast carcinomas. J Clin Oncol. 1999;17:1442-8.

8. Li CI, Uribe DJ, Daling JR. Clinical characteristics of different histologic types of breast cancer. Br J Cancer. 2005;93:1046-52.

9. Louwman MW, Vriezen M, van Beek MW, Nolthenius-Puylaert MC, van der Sangen MJ, Roumen RM, et al. Uncommon breast tumors in perspective: incidence, treatment and survival in the Netherlands. Int J Cancer. 2007;121:127-35.

10. Park S, Koo J, Kim JH, Yang WI, Park BW, Lee KS. Clinicopathological characteristics of mucinous carcinoma of the breast in Korea: comparison with invasive ductal carcinomanot otherwise specified. J Korean Med Sci. 2010;25:361-8.

11. Ranade A, Batra R, Sandhu G, Chitale RA, Balderacchi J. Clinicopathological evaluation of 100 cases of mucinous carcinoma of breast with emphasis on axillary staging and special reference to a micropapillary pattern. J Clin Pathol. 2010;63:10437.
12. Bae SY, Choi MY, Cho DH, Lee JE, Nam SJ, Yang JH. Mucinous carcinoma of the breast in comparison with invasive ductal carcinoma: clinicopathologic characteristics and prognosis. J Breast Cancer. 2011;14:308-13.

13. Hanagiri T, Ono K, Baba T, So T, Yamasaki M, Nagata Y, et al. Clinicopathologic characteristics of mucinous carcinoma of the breast. Int Surg. 2010;95:126-9.

14. Barkley CR, Ligibel JA, Wong JS, Lipsitz S, Smith BL, Golshan M. Mucinous breast carcinoma: a large contemporary series. Am J Surg. 2008;196:549-51.

15. Wu J, Fang Y, Lin L, Fei X, Gao W, Zhu S, et al. Distribution patterns of 21-gene recurrence score in 980 Chinese estrogen receptor-positive, HER2-negative early breast cancer patients. Oncotarget. 2017;8:38706-16.

16. Wang W, Chen X, Lin L, Fei X, Garfield DH, Hong J, et al. Distribution and clinical utility of the 21-gene recurrence score in pure mucinous breast cancer patients: a case-control study. J Cancer. 2018;9:3216-24.

17. Chen X, Zhu S, Fei X, Garfield DH, Wu J, Huang O, et al. Surgery time interval and molecular subtype may influence Ki67 change after core needle biopsy in breast cancer patients. BMC Cancer. 2015;15:822.

18. Zong Y, Zhu L, Wu J, Chen X, Huang O, Fei X, et al. Progesterone receptor status and $\mathrm{Ki}-67$ index may predict early relapse in luminal B/HER2 negative breast cancer patients: a retrospective study. PLoS One. 2014;9:e95629.

19. Hammond ME, Hayes DF, Dowsett M, Allred DC, Hagerty KL, Badve S, et al. American Society of Clinical Oncology / College of American Pathologists guideline recommendations for immunohistochemical testing of estrogen and progesterone receptors in breast cancer. J Clin Oncol. 2010;28:2784-95.

20. Kizy S, Huang JL, Marmor S, Blaes A, Yuan J, Beckwith H, et al. Distribution of 21-gene recurrence scores among breast cancer histologic subtypes. Arch Pathol Lab Med. 2018;142:73541.

21. Gluz O, Nitz UA, Christgen M, Kates RE, Shak S, Clemens M, et al. West German study Group Phase III PlanB Trial: first prospective outcome data for the 21-gene recurrence score assay and concordance of prognostic markers by central and local pathology assessment. J Clin Oncol. 2016;34:2341-9.

22. Di Saverio S, Gutierrez J, Avisar E. A retrospective review with long term follow up of 11,400 cases of pure mucinous breast carcinoma. Breast Cancer Res Treat. 2008;111:541-7. 